\title{
Malignant peripheral nerve sheath tumour of the oesophagus: a case report
}

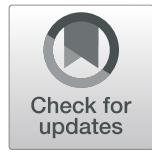

Kento Tomizawa ${ }^{1}$, Tatsuya Miyazaki ${ }^{1 *}$ D, Arisa Yamaguchi ${ }^{1}$, Ryoya Honda ${ }^{1}$, Marie Hoshino ${ }^{1}$, Mitsuhiro Yanai ${ }^{1}$, Yohei Miyamae ${ }^{1}$, Ryo Kurosaki ${ }^{1}$, Hisashi Shimizu' ${ }^{1}$, Kazuhisa Arakawa ${ }^{1}$ and Munenori Ide ${ }^{1,2}$

\begin{abstract}
Background: Malignant peripheral nerve sheath tumour (MPNST) is a very rare disease, and its pathogenesis is unknown. There are few reports of MPNST of the oesophagus. We report a case of an MPNST that was diagnosed and resected.

Case presentation: A 30-year-old female presented with dysphagia. She had been aware of the dysphagia approximately 6 months before presentation. The chest X-ray showed shadows in the right mediastinum. Barium fluoroscopy revealed a semicircular raised lesion in the lower oesophagus. Upper gastrointestinal endoscopy revealed a type 1 oesophageal tumour centred on the posterior wall $26-35 \mathrm{~cm}$ from the incisors. The surface was ulcerated, and the tumour was exposed. The affected area showed no iodine uptake. The EUS showed an isoechoic mass. The $\subset T$ scan showed a mass of $71 \times 61 \times 55$ $\mathrm{mm}$ in the beginning of the lower oesophagus with low density mass and swelling of the right recurrent nerve lymph node to $12 \mathrm{~mm}$. On FDG-PET, the tumour showed an SUVmax of 11.05, and no abnormal accumulation was found in lymph nodes or other organs. The MRI showed a hyperintense mass on the T2Wl, which had prolonged contrast enhancement, and no findings of invasion into surrounding tissue were found. The patient underwent right thoracotomy and open thoracic oesophagectomy. The affected lymph node was tumour negative by rapid pathological diagnosis during the operation. Histologically, spindle cells with different-sized nuclei were mixed throughout the tissue. Some regions showed nuclear polymorphism or a storiform pattern, and locally, there were approximately 7 mitoses/10 HPFs. The margin was relatively clear, but spindle-shaped tumour cells infiltrated the surrounding interstitium and basal myoepithelium, and the patient was diagnosed with MPNST. In this case, the postoperative course was good, and 16 months after the operation, the patient is currently under observation at the outpatient stage without recurrence.
\end{abstract}

Conclusions: MPNST in the oesophagus is a relatively rare disease. Diagnosis before treatment is sometimes difficult, but the prognosis is good if radical resection is possible.

Keywords: FDG-PET, Ki67 index, surgical treatment

\section{Background}

Malignant peripheral nerve sheath tumour (MPNST) is the sixth most common type of soft-tissue sarcoma, accounting for approximately 5 to $10 \%$ of cases [1-3]. Although its exact cellular origins remain unclear, most MPNSTs arise in association with a peripheral nerve and are hypothesized to be of neural crest origin [3, 4]. Gastrointestinal malignant peripheral nerve sheath

\footnotetext{
*Correspondence: tatsuya.miyazaki@maebashi.jr..or.jp

${ }^{1}$ Maebashi Red Cross Hospital Surgery Department, 389-1, Asakura-machi, Maebashi-shi, Gunma 371-0811, Japan

Full list of author information is available at the end of the article
}

tumours are rare, and malignant peripheral nerve sheath tumours of the oesophagus are even rarer. Sarcomas of the oesophagus are rare, representing $0.1-1.5 \%$ of all oesophageal tumours. There are few reports of MPNST of the oesophagus. We report a case of MPNST that was diagnosed and resected.

\section{Case presentation}

A 30-year-old female presented with dysphagia. She was obese, with a height of $157.8 \mathrm{~cm}$ and a weight of $86.8 \mathrm{~kg}$. Her body mass index (BMI) was 34.9.

\section{Springer Open}

(ㅇ The Author(s). 2020 Open Access This article is licensed under a Creative Commons Attribution 4.0 International License, which permits use, sharing, adaptation, distribution and reproduction in any medium or format, as long as you give appropriate credit to the original author(s) and the source, provide a link to the Creative Commons licence, and indicate if changes were made. The images or other third party material in this article are included in the article's Creative Commons licence, unless indicated otherwise in a credit line to the material. If material is not included in the article's Creative Commons licence and your intended use is not permitted by statutory regulation or exceeds the permitted use, you will need to obtain permission directly from the copyright holder. To view a copy of this licence, visit http://creativecommons.org/licenses/by/4.0/. 
A

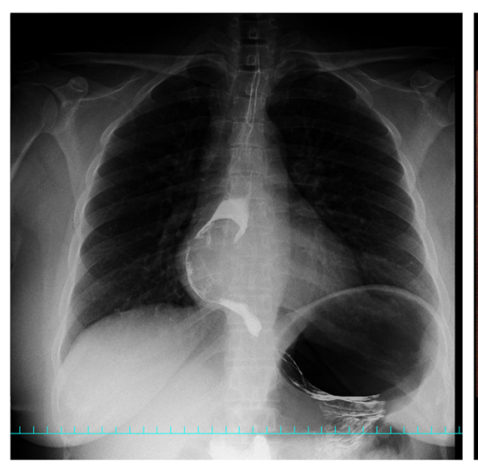

B

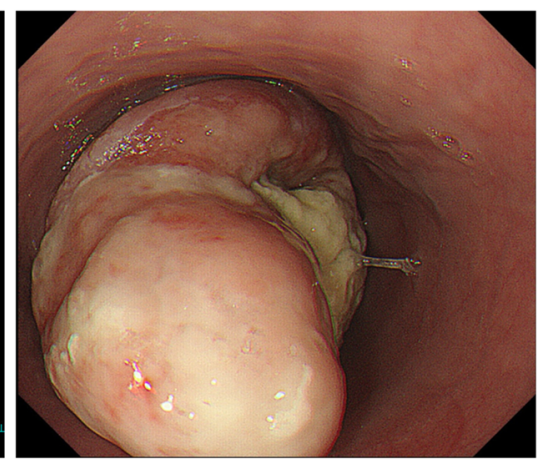

Fig. 1 a Upper gastrointestinal endoscopy revealed a type 1 oesophageal tumour centred on the posterior wall $26-35 \mathrm{~cm}$ from the incisors. The surface was ulcerated, and the tumour was exposed. b Upper gastrointestinal imaging revealed a semicircular raised lesion in the lower oesophagus

She had been aware of the dysphagia for approximately 6 months before presentation. During a medical check-up for work, a chest X-ray showed a shadow on the mediastinum, so the patient visited a doctor. The chest CT showed a 55-mm circular shadow. Three years ago, an upper GI endoscopy revealed a raised lesion in the left anterior wall of the oesophagus. At that time, the lesion was $1 / 3$ of a circle and approximately $25 \mathrm{~mm}$ in size. This lesion was diagnosed as leiomyoma and was monitored. The patient had no medical history and no specific mention of a family medical history.

Blood tests showed a slight increase in white blood cell count and CRP. CEA, CA 19-9, NSE, SCC, and p53 levels were within the reference ranges. The chest X-ray showed shadows in the right mediastinum (Fig. 1a). Barium fluoroscopy revealed a semicircular raised lesion in
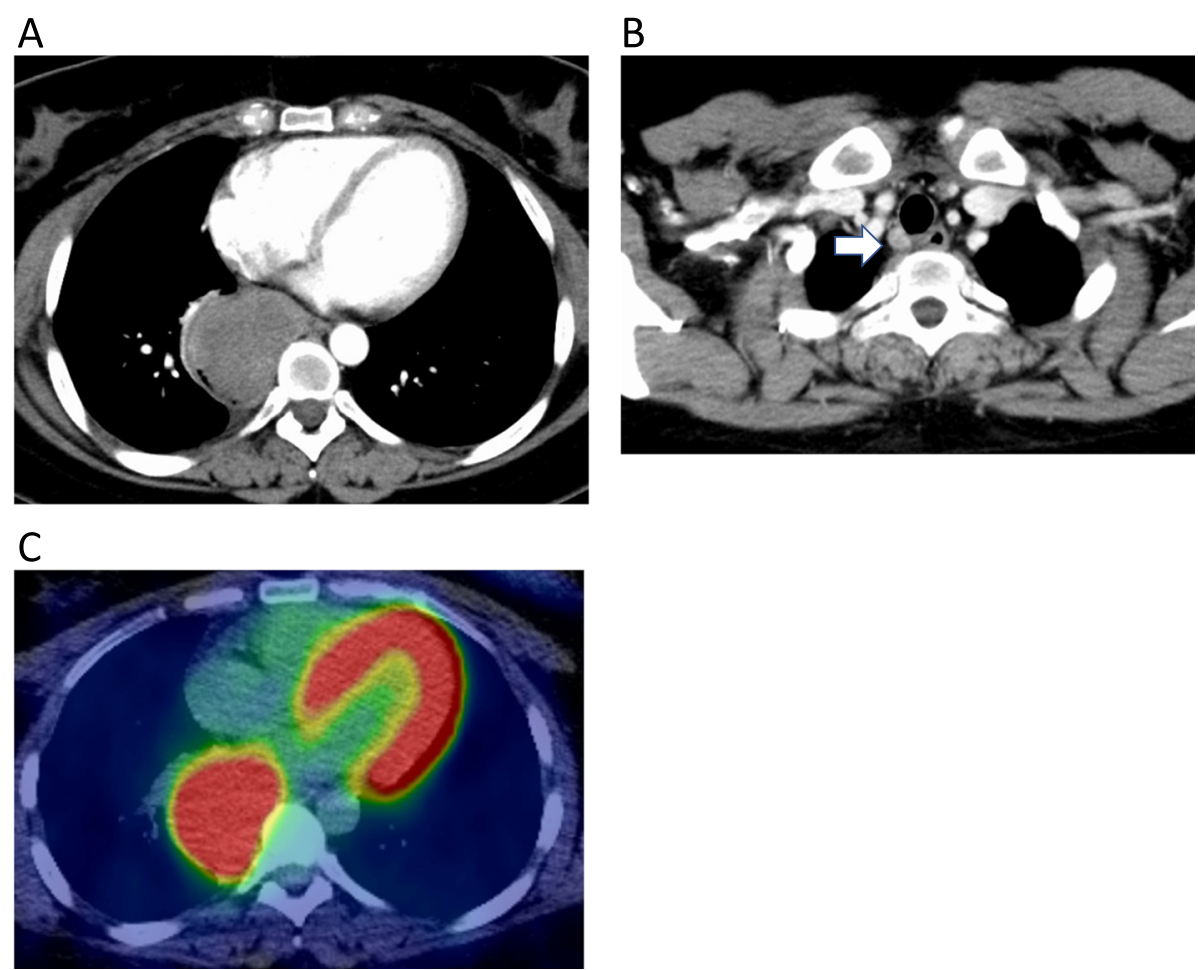

Fig. 2 a The $C T$ scan showed a mass of $71 \times 61 \times 55 \mathrm{~mm}$ in the beginning of the lower oesophagus with poor differentiation and progressive contrast enhancement. $\mathbf{b}$ The white arrow indicates swelling of up to $12 \mathrm{~mm}$ of the right recurrent nerve lymph node on the $\subset T$ scan. c On FDG-PET, the tumour showed an SUVmax of 11.05, and no abnormal accumulation was found in lymph nodes or other organs. FDG-PET, 18F-fluorodeoxyglucose positron emission tomography 
the lower oesophagus (Fig. 1a). Upper gastrointestinal endoscopy revealed a type 1 oesophageal tumour centred on the posterior wall $26-35 \mathrm{~cm}$ from the incisors (Fig. $1 \mathrm{~b})$. The surface was ulcerated, and the tumour was exposed (Fig. 1b). The tumour was hard and white. The affected area showed no iodine uptake. The EUS showed an isoechoic mass. The CT scan showed a mass of $71 \times$ $61 \times 55 \mathrm{~mm}$ in the beginning of the lower oesophagus with low density mass (Fig. 2a) and swelling of the right recurrent nerve lymph node to $12 \mathrm{~mm}$ (Fig. 2b). On FDG-PET, the tumour showed an SUVmax of 11.05, and no abnormal accumulation was found in lymph nodes or other organs (Fig. 2c). The MRI showed a hyperintense mass on the T2WI, which had prolonged contrast enhancement, and no findings of invasion into surrounding tissue were found. The endoscopic biopsy showed cascading spindle cells with different-sized nuclei (Fig. 3a), and immunostaining showed S-100 (+) (Fig. 3b), keratin $(-)$, CD34 (-), and desmin (-) staining. The MIB-1 LI was approximately $25 \%$ (Fig. 3c), which led to the diagnosis of malignant schwannoma.

We performed right thoracotomy and open thoracic oesophagectomy. There were no pleural effusions or adhesions in the pleural cavity. The swollen right recurrent nerve lymph node was negative for tumours by rapid pathological diagnosis during the operation. We dissected all of the thoracic oesophagus with tumours to achieve radical resection. We placed a gastric tube through the intrathoracic route for reconstruction. Intrathoracic oesophagogastric anastomosis was performed using the circular stapling technique. The resected tumour was white, hard, and $75 \times 45 \times 45 \mathrm{~mm}$ in size (Fig. 4). A large bulging lesion in the neck with degenerative necrosis was observed in the specimen. The raised part of the tumour was covered with normal mucosa (Fig. 4c).

Histologically, spindle cells with different-sized nuclei were mixed throughout the tissue (Fig. 5a). Some regions showed nuclear polymorphism or a storiform pattern, and locally, there were approximately 7 mitoses/10 HPFs (Fig. 5b). The MIB-1 LI was approximately $25 \%$ (Fig. 5 c). The margin was relatively clear, but spindle-shaped tumour cells infiltrated the surrounding interstitium and basal myoepithelium, and the patient was diagnosed with MPNST (Fig. 5a, b). Immunostaining showed S-100 (+) (Fig. 5d), CD34 (-), and HMB-45 (-) staining. These results led to a diagnosis of MPNST. In this case, the postoperative course was good, and 16 months after the operation, the patient is currently under observation at the outpatient stage without recurrence.

\section{Discussion}

In PubMed, a medical publication database, there are 16 case reports of oesophageal MPNST, including this case
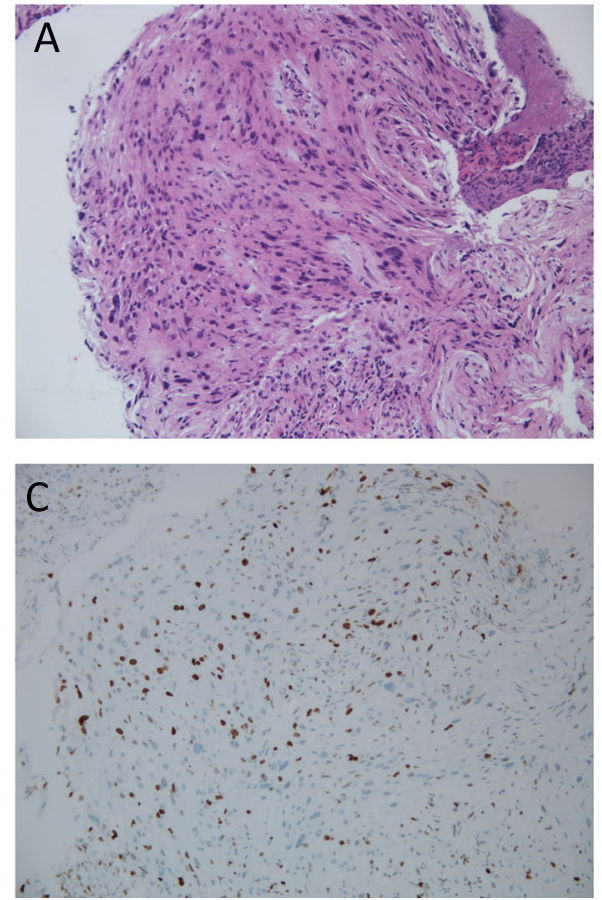

MIB-1 LI:25\%

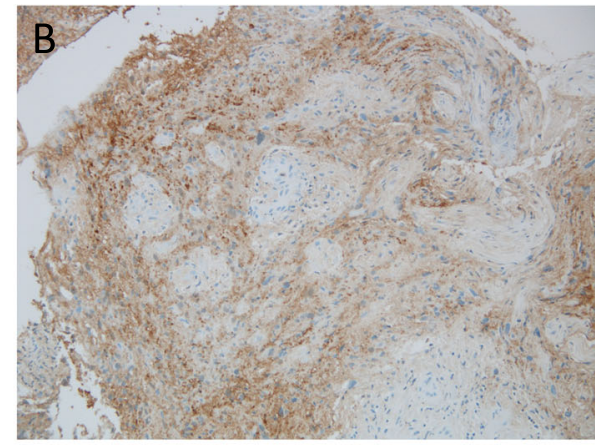

S-100 positive

Fig. 3 a Histopathological findings of the biopsy sample. The pathological specimen revealed that spindle cells with different-sized nuclei were mixed throughout the tissue. $\mathbf{b}$ Immunostaining showed S-100-positive staining. c Immunostaining showed MIB-1-positive staining. The MIB-1 LI was approximately $25 \%$ 


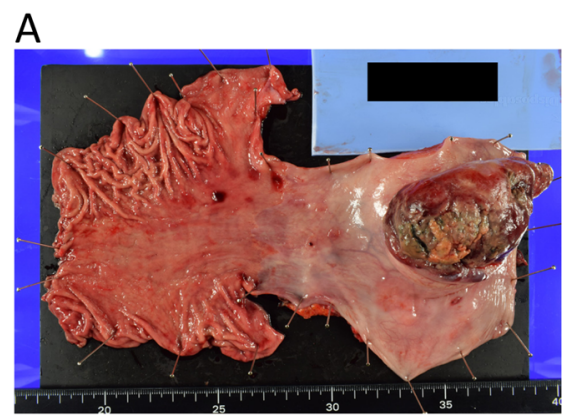

C

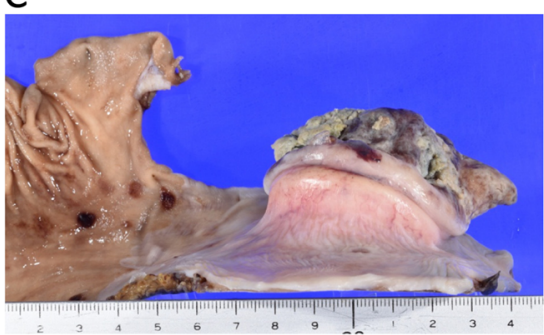

B

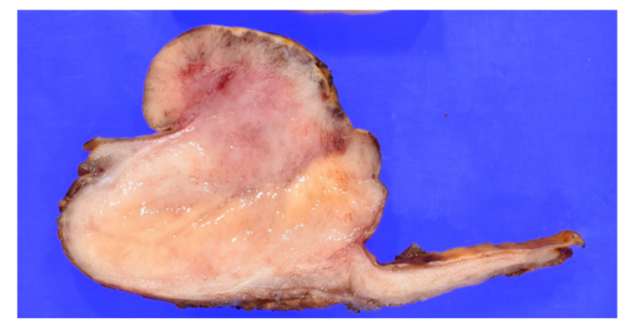

Fig. 4 a Macroscopic findings. The resected tumour was $75 \times 45 \times 45 \mathrm{~mm}$ in size. b A large bulging lesion with a degenerative necrosis was observed in the neck. $\mathbf{c}$ The raised part of the tumour was covered with normal mucosa.
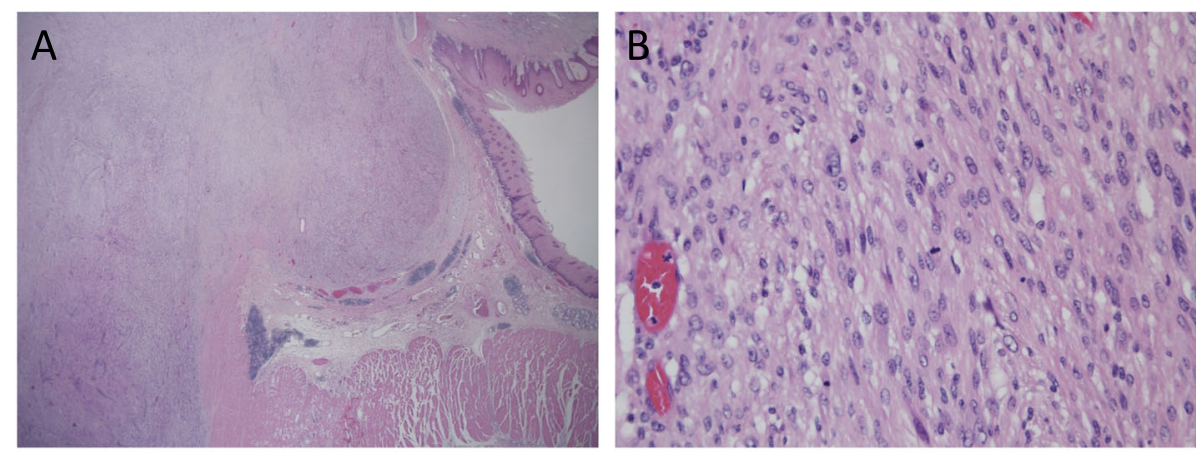

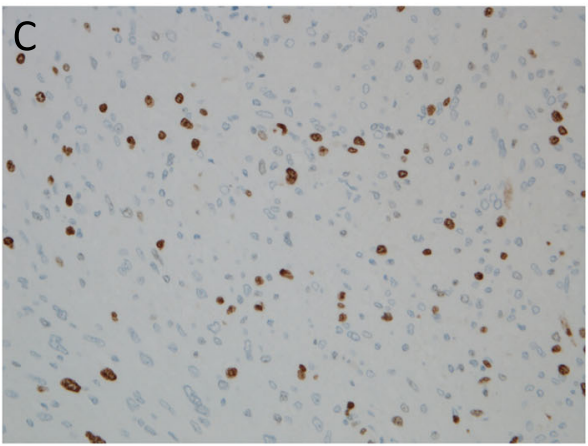

MIB-1 LI:25\%

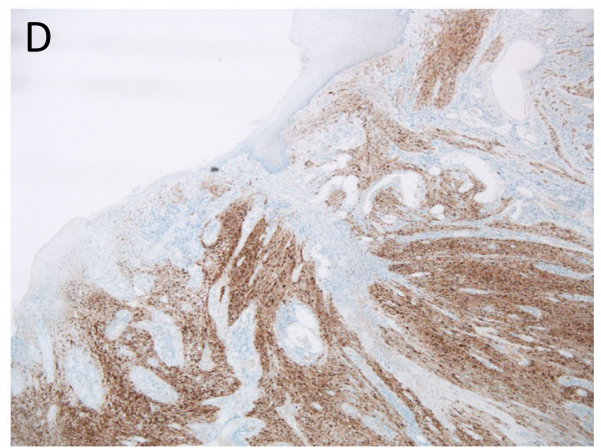

S-100 positive

Fig. 5 a, b Histopathological findings. The pathological specimen revealed that spindle cells with different-sized nuclei were mixed throughout the tissue. Some regions showed nuclear polymorphism or a storiform pattern, and locally, there were approximately 7 mitoses/10 HPFs. The margin was relatively clear, but spindle-shaped tumour cells infiltrated the surrounding interstitium and basal myoepithelium, and the patient was diagnosed with malignant peripheral nerve sheath tumour (MPNST). c Immunostaining showed MIB-1-positive staining. The MIB-1 LI was approximately 25\%. d Immunostaining showed S-100-positive staining. 
[5-11] (Table 1). The onset age was 27-76 years, with an average of 50.0 years. More people had a young age of onset than an older age of onset. MPNST often also occurs in adults aged 20 to 50 years in other organs. The sex ratio was 4 to 12 in favour of women. The tumour diameter ranged from 2.5 to $17 \mathrm{~cm}$. Our case was a young woman with a tumour diameter of $7.1 \mathrm{~cm}$ who had more of the common characteristics than the reported cases. Among the 16 cases, only four patients were diagnosed with MPNST before treatment. MPNST may be difficult to diagnose because the shape of the tumour resembles that of a submucosal tumour, which is also similar to oesophageal schwannoma; thus, MPNS $\mathrm{T}$ is usually undiagnosable by routine biopsy. EUSFNAB (endoscopic ultrasound-guided fine needle aspiration biopsy) and boring biopsy are useful for the diagnosis of submucosal tumours. In our case, because this patient had ulcer on the top of the protruding tumour, biopsy and diagnosis were easy. The macroscopic features of two cases were ulcers, and our patients had ulcers on top of the protruding type. The two patients with ulcers were diagnosed with MPNST, and one patient was diagnosed with a malignant tumour. In our case, we collected 6 biopsy samples from the tumour surface, around the ulcer and floor. Appropriate amounts of tumour cells were found only from the biopsy of the ulcer floor. The biopsies from the tumour surface detected necrotic tissue and fibroblasts. If we could not obtain an accurate diagnosis from the first biopsy, we would need a boring biopsy or EUS-FNAB. In these series, 8 patients underwent enucleation, and 7 patients underwent oesophagectomy. Because our patient was diagnosed with malignant disease before surgery, oesophagectomy was performed for to achieve radical resection. In this series of MPNSTs in the oesophagus, there was no recurrence in patients who underwent resection, including enucleation. However, many cases of local recurrence of MPNST in other organs have been reported. Nonetheless, when possible, we think that MPNST of the oesophagus should also achieve perfect local control. Two cases had distant metastasis, and these cases had a poor prognosis. On the other hand, the prognosis was good in cases in which radical resection was possible. Therefore, cases with distant metastases may require systemic treatment, such as chemotherapy. Few drugs have been shown to be effective, and treatment comprises the single agent doxorubicin or a combination of doxorubicin and ifosfamide [12]. Furthermore, with regard to malignancy, nerve cells normally abundantly express glucose transporter type 3 (GLUT-3), which transports for glucose into cells, and show high uptake during FDG-PET. It is difficult to evaluate the grade of malignancy from preoperative imaging tests. MPNST has an MIB-1 LI of 5 to $65 \%$, whereas the MIB-1 LI for schwannoma is often $1 \%$ or less, which is useful for differentiation of benign versus malignant lesions.

Patients with neurofibromatosis type 1 have an approximately $10 \%$ probability of developing MPNST during their lifetime. In addition, approximately $50 \%$ of MPNSTs develop in patients with neurofibromatosis type 1. Treatment is based on extensive resection. Recently, a large cohort study showed that radiotherapy and chemotherapy were not associated with survival [13]. The treatment benefits of radiotherapy and chemotherapy for MPNST are unclear and controversial. MPNST is less responsive to radiation and chemotherapy, and surgical resection is central to its treatment. In addition, chemotherapy is indicated for large lesions and metastases. The most frequent sites of distant metastasis are the lungs, followed by the bones and the lymph nodes in the bones. Neurofibromatosis type 1 is not considered to be a risk factor involved in prognosis.

\section{Conclusion}

Primary oesophageal MPNST is a relatively rare disease. Diagnosis before treatment is sometimes difficult, but the prognosis is good if radical resection is possible.

\section{Abbreviations \\ MPNST: Malignant peripheral nerve sheath tumour; EUS: Endoscopic ultrasonography; CT: Computed tomography; FDG-PET: 18F-fluorodeoxy glucose-positron emission tomography; SUV: Standardized uptake value; HPFs: High power fields; EUS-FNAB: Endoscopic ultrasound-guided fine nee- dle aspiration biopsy}

\section{Acknowledgements \\ None}

\section{Authors' contributions}

KT reported the case and wrote the manuscript. AY, $\mathrm{RH}, \mathrm{MH}, \mathrm{MY}, \mathrm{YM}, \mathrm{RK}, \mathrm{HS}$, and KA helped to draft the manuscript. TM participated in revising the manuscript critically. Ml investigated pathological study and diagnosis. All authors declare that they contributed to this article and that they have read and approved the final manuscript.

\section{Authors' information}

None

\section{Funding}

No grant support or funding from public institutions or private enterprises was received.

Availability of data and materials

Not applicable because this manuscript is a case report.

Consent for publication

Written informed consent was obtained from the patient for the publication of this case report and the accompanying images.

\section{Competing interests}

The authors declare that they have no competing interests.

\section{Author details}

'Maebashi Red Cross Hospital Surgery Department, 389-1, Asakura-machi, Maebashi-shi, Gunma 371-0811, Japan. ${ }^{2}$ Maebashi Red Cross Hospital Pathology Department, 389-1, Asakura-machi, Maebashi-shi, Gunma 371-0811, Japan. 


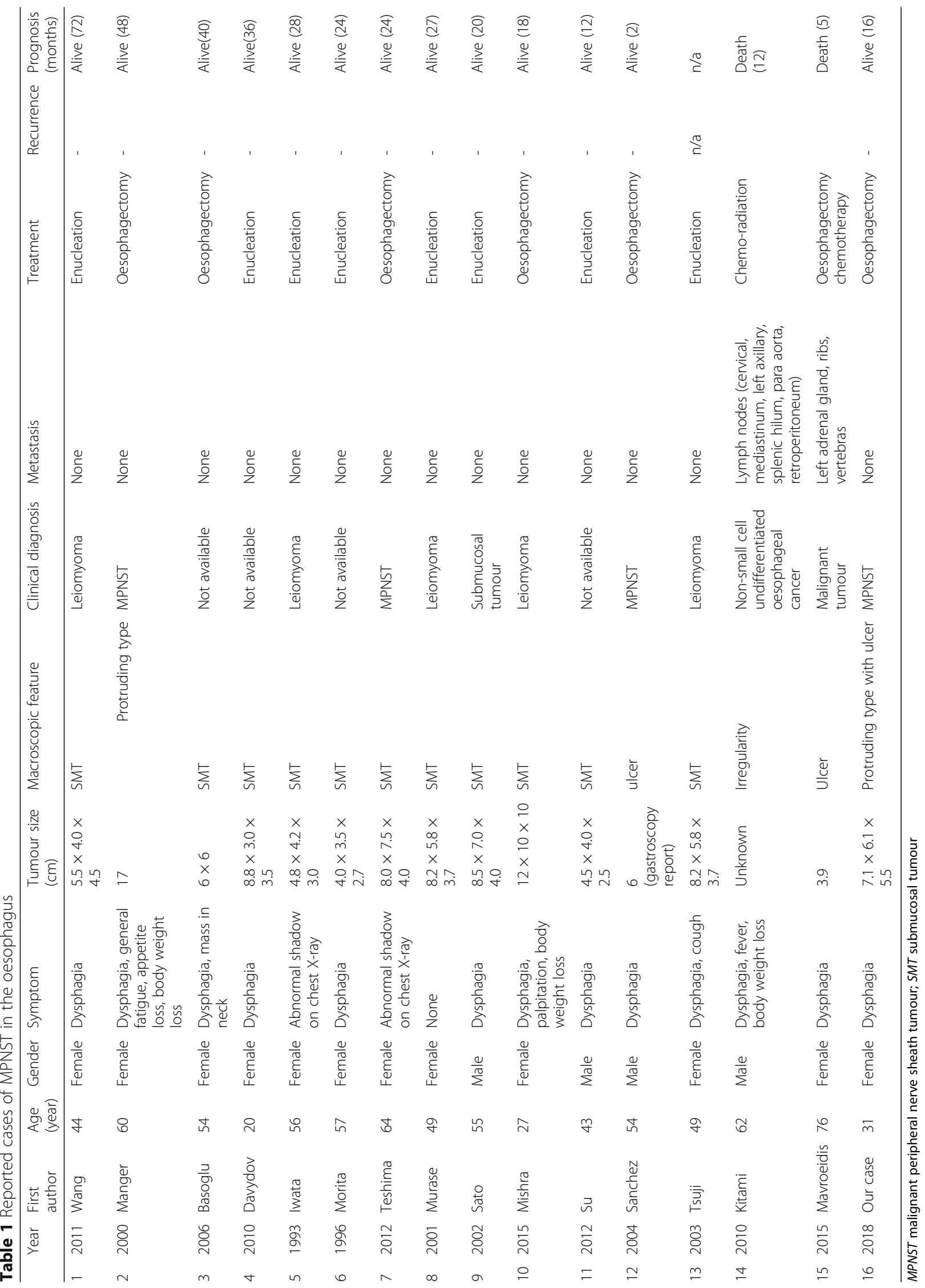


Received: 16 March 2020 Accepted: 22 July 2020

Published online: 31 July 2020

\section{References}

1. Eilber FC, Brennan MF, Eilber FR, et al. Validation of the postoperative nomogram for 12-year sarcoma-specific mortality. Cancer. 2004;101(10): 2270-5.

2. Grobmyer SR, Reith JD, Shahlaee A, et al. Malignant peripheral nerve sheath tumor: molecular pathogenesis and current management considerations. J Surg Oncol. 2008;97(4):340-9.

3. James AW, Shurell E, Singh A, et al. Malignant peripheral nerve sheath tumor. Surg Oncol Clin N Am. 2016;25:789-802.

4. Lin CT, Huang TW, Nieh S, et al. Treatment of a malignant peripheral nerve sheath tumor. Onkologie. 2009;32(8-9):503-5.

5. Wang $S$, Zheng J, Zheng $R$, et al. Long-term survival in a rare case of malignant esophageal schwannoma cured by surgical excision. Ann Thorac Surg. 2011;92:357-8.

6. Manger T, Pross M, Haeckel C, et al. Malignant peripheral nerve sheath tumor of the esophagus. Dig Surg. 2000;17:627-31.

7. Murase K, Hino A, Ozeki Y, et al. Malignant schwannoma of the esophagus with lymph node metastasis: literature review of schwannoma of the esophagus. J Gastroenterol. 2001:36(11):772-7.

8. Sato N, Sato T, Sato K, et al. A resected case of malignant schwannoma of the esophagus. Surgery. 2002;56(10):1553-7.

9. Mishra B, Madhusudhan KS, Kilambi R, et al. Malignant schwannoma of the esophagus: a rare case report. Kor J Thorac Cardiovasc Surg. 2016:49(1):63-6.

10. Kitami M, Ono K, Nakata $H$, et al. A case of esophageal malignant schwannoma. Ainikai Med Res J. 2010;41:85-8.

11. Mavroeidis VK, Kandilaris K, Matthioudakis DI, et al. Malignant peripheral nerve sheath tumor of the oesophagus Review of the literature and report of a case with lymph node and distant metastases. Ann Ital Chir. 2017:88: 26-38.

12. Santoro A, Tursz T, Mouridsen $H_{\text {, et }}$ al. Doxorubicin versus CYVADIC versus doxorubicin plus ifosfamide in first-line treatment of advanced soft tissue sarcomas: a randomized study of the European Organization for Research and Treatment of Cancer Soft Tissue and Bone Sarcoma Group. J Clin Oncol. 1995:13(7):1537-45.

13. Martin E, Coert JH, Flucke UE, et al. A nationwide cohort study on treatment and survival in patients with malignant peripheral nerve sheath tumours. Eur J Cancer. 2020;124:77-87.

\section{Publisher's Note}

Springer Nature remains neutral with regard to jurisdictional claims in published maps and institutional affiliations.

\section{Submit your manuscript to a SpringerOpen ${ }^{\circ}$ journal and benefit from:}

- Convenient online submission

- Rigorous peer review

- Open access: articles freely available online

- High visibility within the field

- Retaining the copyright to your article

Submit your next manuscript at $\boldsymbol{\nabla}$ springeropen.com 